\title{
Revised manuscript
}

Insights into the effects of $\mathrm{N}$-glycosylation on the characteristics of the VC1 domain of the human receptor for advanced glycation end products (RAGEs) secreted by Pichia pastoris

\author{
Glycoconjugate Journal
}

Genny Degani ${ }^{1}$, Alberto Barbiroli ${ }^{2}$, Paula Magnelli ${ }^{3}$, Stefania Digiovanni ${ }^{1}$, Alessandra Altomare ${ }^{4}$, Giancarlo Aldini ${ }^{4}$ and Laura Popolo ${ }^{1 *}$

${ }^{1}$ Department of Biosciences, Via Celoria 26, and ${ }^{2}$ Department of Food, Environmental and Nutritional Sciences (DeFENS) University of Milan, 20133 Milan, Italy

${ }^{3}$ New England Biolabs, Inc., Ipswich, MA, 01938, USA

${ }^{4}$ Department of Pharmaceutical Sciences (DISFARM), Via Mangiagalli 25, University of Milan

genny.degani@unimi.it

alberto.barbiroli@unimi.it

Magnelli@neb.com

stefania.digiovanni@unimi.it

alessandra.altomare@unimi.it

giancarlo.aldini@unimi.it

*Corresponding author

Laura Popolo

University of Milan

Department of Biosciences

Via Celoria 26

20133 Milano

Italy

Tel: +39-02-50314919

E-mail address: laura.popolo@unimi.it 


\begin{abstract}
Advanced glycation end products (AGEs) and advanced lipoxidation end products (ALEs), resulting from non-enzymatic modifications of proteins, are potentially harmful to human health. They directly act on proteins, affecting structure and function, or through receptor-mediated mechanisms. RAGE, a type I transmembrane glycoprotein, was identified as a receptor for AGEs. RAGE is involved in chronic inflammation, oxidative stress-based diseases and ageing. The majority of RAGE ligands bind to the VC1 domain. This domain was successfully expressed and secreted by Pichia pastoris. Out of two $N$-glycosylation sites, one (Asn25) was fully occupied while the other (Asn81) was under-glycosylated, generating two VC1 variants, named p36 and p34. Analysis of $N$-glycans and of their influence on VC1 properties were here investigated.

The highly sensitive procainamide labeling method coupled to ES-MS was used for $N$-glycan profiling. $\mathrm{N}$-glycans released from VC1 ranged from $\mathrm{Man}_{9} \mathrm{GlcNAc}_{2}$ - to $\mathrm{Man}_{15} \mathrm{GlcNAc}_{2}$ - with major Man $_{10} \mathrm{GlcNAc}_{2}$ - and $\mathrm{Man}_{11} \mathrm{GlcNAc}_{2}$ - species for p36 and p34, respectively. Circular dichroism spectra indicated that VC1 maintains the same conformation also after removal of $N$-glycans. Thermal denaturation curves showed that the carbohydrate moiety has a small stabilizing effect on VC1 protein conformation. The removal of the glycan moiety did not affect the binding of VC1 to sugar-derived AGE- or malondialdehyde-derived ALE-human serum albumin.

Given the crucial role of RAGE in human pathologies, the features of VC1 from P. pastoris will prove useful in designing strategies for the enrichment of AGEs/ALEs from plasma, urine or tissues, and in characterizing the nature of the interaction.
\end{abstract}

Keywords: Receptor for Advanced Glycation End products (RAGE) - Protein glycoforms Released glycan profiling - LC/Mass spectrometry - Thermal stability - protein-protein interactions - Pichia pastoris 


\section{Introduction}

Reactive carbonyl species (RCS) are breakdown products arising from the oxidation of sugars and lipids. RCS are chemically quite heterogeneous, belonging to different classes including dialdehydes [glyoxal and malondialdehyde (MDA)], keto-aldehydes (methylglyoxal) and $\alpha, \beta$ unsaturated aldehydes [hydroxynonenal and acrolein]. RCS species covalently react with nucleophilic substrates such as proteins, leading to the formation of covalent adducts named advanced glycation end products (AGEs) when RCS originate from sugars and advanced lipoxidation end products (ALEs) when RCS originate from lipids [1, 2]. AGEs/ALEs are involved in the initiation and progression of different human diseases including type II diabetes, cardiovascular diseases, nephropathies and neurodegenerative diseases ([3] and see the reviews [46] in the Special Issue on Advanced Glycation in Diabetes, Aging and Age-related Diseases in Glyconj. J. vol. 33, issue 4 (2016)).

The damaging effect of AGEs/ALEs is mediated by different mechanisms, including the dysfunction of proteins by oxidative modifications, protein polymerization and cross-linking. Relevant examples are provided by long-lived proteins, such as lens proteins or collagens that accumulate AGEs in conditions of chronic inflammation and during ageing affecting organ and tissue functions [7-9]. Another mechanism involves signal transduction pathways, and in particular the pathways mediated by RAGE, first identified as a surface receptor for AGEs [10, 11]. Signaling cascades induced by RAGE binding (the AGEs-ALEs/RAGE damaging axis) activate the transcription nuclear factor-kappa $\mathrm{B}(\mathrm{NF}-\mathrm{\kappa B})$, leading to an increased release of inflammatory cytokines such as TNF- $\alpha$ and the formation of reactive oxygen species (ROS), which further increase cellular oxidative damage $[2,12]$. The AGEs-ALEs/RAGE damaging axis is a promising drug target and some molecular approaches, involving RAGE antagonists as well as AGEs and ALEs inhibitors, have been proposed [13]. Other RAGE ligands are: heparan sulfate and chondroitin sulfate (that also induce RAGE oligomerization in the plane of the membrane) $[14,15]$, S100 proteins, High-Mobility Group protein Box-1 (HMGB1) and amyloid- $\beta$ peptides. Most of the ligands share a negatively charged surface that interacts with the highly positive $\mathrm{V}$ domain of RAGE.

Although several advancements have been made in this field in recent years, one main undiscovered aspect involves the identification and characterization of AGEs and ALEs as RAGE activators. Characterization of circulating AGEs and ALEs is an analytical challenge not only because of their 
heterogeneity but also because they are formed in negligible amounts with respect to the unmodified proteins [16].

In order to enrich AGEs and ALEs from biological sources, we have recently proposed an affinity strategy based on the use of RAGE, a type I transmembrane glycoprotein [17]. The extracellular portion is composed of a V-type immunoglobulin (Ig) domain (V) and two C-type Ig domains (C1 and C2). Notably, V and C1 form a single folding unit to which the majority of RAGE ligands bind [18-20]. VC1 of human RAGE (hRAGE) was immobilized to an insoluble matrix and its capability to capture model AGEs and ALEs also in simulated complex biological liquids, such as milk or serum, was validated [17]. In our studies, we use VC1 secreted as a glycosylated protein form Pichia pastoris since it is remarkably more stable and soluble compared to the unmodified counterpart produced in E. coli [19].

VC1 has two $N$-glycosylation sites (Asn25 and Asn81) both present in the V portion. RAGE is unique to mammals and the two $N$-glycosylation sequons (25-NIT and 81-NGS) are conserved in all mammalian RAGE sequences we analyzed. In a previous peptide mapping analysis, we showed that in $P$. pastoris $N$-glycan site occupancy of VC1 was full at Asn25 and partial at Asn81 [19]. Similar results were reported for the entire extracellular portion of RAGE (sRAGE) from P. pastoris or for membrane-bound RAGE expressed in mammalian cell lines [21, 22]. In addition, the residue Asn81 also undergoes spontaneous deamidation and this is promoted by the presence of Gly in the second position of the sequon via cyclization of asparagine by a $\beta$-aspartyl shift mechanism $[19,22,23]$.

To further characterize the $N$-glycosylation of the single VC1 domain, in this work we fully analyzed the $N$-glycans of the recombinant VC1 secreted by $P$. pastoris. Since the importance of $N$ glycosylation for the biological or the enzymatic activity and for protein stability is specific for each glycoprotein, we tested the role of $N$-glycosylation on the conformation stability and the possible implications on VC1 function. The study focused on sugar-derived AGEs but the binding tests were extended to ALEs following our recent in vitro demonstration that VC1 binds to ALEs derived from malondialdehyde treatments of human serum albumin (HSA) [17].

\section{Materials and methods}

\subsection{Strain, growth conditions and induction of protein expression}

The recombinant $P$. pastoris KM71 strain (Mut ${ }^{\mathrm{S}}, \mathrm{Arg}^{+}$, His') (Invitrogen) expressing VC1-His tagStrep tag, was previously described [17]. To induce the expression of the recombinant VC1, $P$. pastoris growing cells were shifted from a culture in Glycerol-complex Medium (MGY) (1\% yeast 
extract, $2 \%$ peptone, $1 \%$ glycerol, $1.34 \%$ YNB, $4 \times 10^{-5} \%$ biotin) to a mixed Methanol-Ethanol complex Medium (MEMY) (1\% yeast extract, 2\% peptone, 0.5\% methanol, 0.7\% ethanol, 1.34\% $\mathrm{YNB}, 4 \times 10^{-5} \%$ biotin) at an initial $\mathrm{OD}_{600 \mathrm{~nm}}$ of $\sim 1$. Cells were routinely grown in flasks at $30{ }^{\circ} \mathrm{C}$ under strong agitation and growth was monitored through the increase in optical density at $600 \mathrm{~nm}$ $\left(\mathrm{OD}_{600}\right)$. Methanol was added daily to a $0.5 \%(\mathrm{v} / \mathrm{v})$ final concentration. The culture supernatant was collected $72 \mathrm{~h}$ after induction $\left(\mathrm{OD}_{600}\right.$ about 30) by centrifugation of the culture at 4,300 x g for 15 min at $4{ }^{\circ} \mathrm{C}$. The culture supernatant was filtered (Nitrocellulose $0.22 \mu \mathrm{m}$ ) and either frozen at -20 ${ }^{\circ} \mathrm{C}$ or immediately processed.

\subsection{Purification of p34 and p36 glycoforms of VC1}

The VC1 domain was purified from the culture supernatant by Nickel affinity chromatography and to separate the 34-kDa (p34) and 36-kDa (p36) forms, a modified procedure of a previously published protocol was adopted [17]. Briefly, $1.5 \mathrm{mg}$ of purified VC1 was dialyzed (Spectra/Por membranes, cut-off 6-8,000) at $4{ }^{\circ} \mathrm{C}$ for $16 \mathrm{~h}$ against $10 \mathrm{mM}$ Na-acetate $\mathrm{pH}$ 5.0. The sample was applied to a cation-exchange HI-TRAP SP HP column (volume $2 \mathrm{~mL}$, GE Healthcare) connected to an ÄKTA-Prime plus system (GE Healthcare) and equilibrated with $10 \mathrm{mM}$ Na-acetate pH 5.0. The column was washed with the same buffer until the $A_{280}$ reached the baseline. p34 and p36 were eluted applying a linear ionic strength gradient of $10 \mathrm{mM}$ Na-acetate $\mathrm{pH} 5.0$ containing $1 \mathrm{M} \mathrm{NaCl}$ (Fig. S1). The separation of p34 and p36 was confirmed by SDS polyacrylamide gel electrophoresis (SDS-PAGE). Purified p34 and p36 were applied to an Amicon Ultra- diafiltration system (0.5 mL, cut off 10K, Millipore) and buffer was changed to $10 \mathrm{mM} \mathrm{Na}$-acetate, $25 \mathrm{mM} \mathrm{NaCl}$, pH 5.0 for the

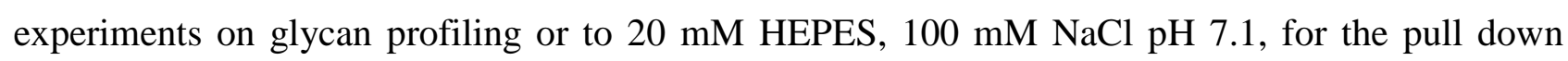
assays.

\section{3 p34 and p36 N-glycan profiling}

Purified p34 and p36 proteins (about $500 \mu$ g each) were lyophilized and stored at $-20{ }^{\circ} \mathrm{C}$. After reconstitution in $100 \mu \mathrm{L}$ of water, two $20 \mu \mathrm{L}$ aliquots were deglycosylated with Rapid PNGase F (NEB \#P0710) following manufacturer's instructions (2-step protocol that includes a pre-incubation at $80^{\circ} \mathrm{C}$ for $2 \mathrm{~min}$ in Rapid PNGase buffer for complete deglycosylation). Released $N$-glycans were labeled with procainamide [4-amino-N-(2-diethylaminoethyl) benzamide] according to a previously described procedure [24]. 
Samples were analyzed using a BEH-XBridge column, (130 A, $2.5 \mu \mathrm{m}, 2.1 \mathrm{~mm}$ x $150 \mathrm{~mm}$; Waters) on a Dionex UltiMate ${ }^{\circledR}$ HPLC with fluorescent detection, in line with a LTQ ${ }^{\mathrm{TM}}$ Orbitrap Velos ${ }^{\mathrm{TM}}$ Spectrometer (HESI-II probe), using optimized settings for positive mode detection as described before [24]. Structures were assigned based on retention time, $\mathrm{m} / \mathrm{z}$ (parent ion), and in accordance with known biosynthetic pathways. Fluorescently labeled $\mathrm{N}$-glycans standards ( $\mathrm{Man}_{9} \mathrm{GlcNac}_{2}$ to $\mathrm{Man}_{3} \mathrm{GlcNac}_{2}$ series) were used for calibration. Sugar composition of the core chains was assessed by use of a recombinant Jack Bean mannosidase. About $0.5 \mathrm{nmol}$ of total VC1 N-glycans were subjected to enzymatic treatment with 2 units of $\alpha-1,2,3,6$ Mannosidase (NEB \#P0768), in a total

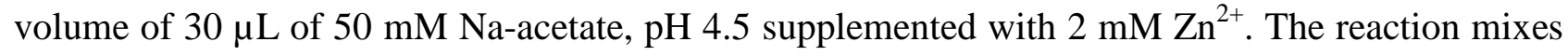
were incubated for $4 \mathrm{~h}$ at $37^{\circ} \mathrm{C}$. Samples were analyzed by liquid chromatography and mass spectrometry as described above.

\subsection{Endo H treatment of VC1 in native conditions}

To remove the $N$-linked oligosaccharide chains from VC1 in native conditions, we used Endoglycosidase H (Endo H). The reaction mix containing $60 \mu \mathrm{g}$ of purified VC1 in $50 \mathrm{mM}$ sodium acetate buffer pH 6 and $5000 \mathrm{U}$ of Endo H (NEB \#P0702) was incubated for 16 hours at $37{ }^{\circ} \mathrm{C}$. Untreated VC1 (not containing Endo $\mathrm{H}$ ) was incubated in parallel. The removal of $N$-glycans was verified by SDS-PAGE using anykD pre-cast minigels (Bio-Rad) and Biosafe Coomassie staining (Bio-Rad). Endo H-treated VC1 was diluted to $0.3 \mathrm{mg} / \mathrm{mL}$ and adjusted to $\mathrm{pH} 7.1$ by addition of HEPES Buffer (20 mM HEPES pH 7.4, $100 \mathrm{mM} \mathrm{NaCl)}$ and used for immobilization on magnetic beads.

\subsection{Circular dichroism}

Circular dichroism (CD) studies were carried out on a J-810 spectropolarimeter (JASCO Europe, Cremella, Italy) equipped with a Peltier system for temperature control. All measurements were performed in a $0.1 \mathrm{~cm}$ path length cuvette, at $0.2 \mathrm{mg} / \mathrm{mL}$ protein concentration, in $50 \mathrm{mM}$ sodium phosphate, $\mathrm{pH}$ 7.1. Far-UV spectra were recorded from 195 to $260 \mathrm{~nm}$ at $20^{\circ} \mathrm{C}$. Temperature ramps were recorded from 20 to $95{ }^{\circ} \mathrm{C}$ at a heating rate of $1{ }^{\circ} \mathrm{C} / \mathrm{min}$, while continuously monitoring the change of CD signal at $205 \mathrm{~nm}$. $T_{m}$ values were determined as the maximum of the first derivative of the unfolding profiles.

2.6 Pull down assay with VC1, Endo H-treated VC1 and p34/p36 
Endo H-untreated and -treated VC1, p34 and p36 were immobilized in equal amount through their C-terminal Strep Tag to Streptavidin Mag Sepharose beads (GE Healthcare) as previously described [17] to obtain untreated-VC1-beads, Endo H-treated-VC1-beads, p34 or p36-beads. As control matrices, native VC1, not incubated at $37{ }^{\circ} \mathrm{C}$, immobilized on Streptavidin Mag Sepharose (VC1beads), or Streptavidin Mag Sepharose alone (control beads) were used.

AGE and ALE-human serum albumin (HSA) were obtained by incubation of recombinant HSA expressed in $P$. pastoris (Sigma, Cat no. A7736) with ribose or malondialdehyde (MDA) as previously described [17]. The beads were incubated for $1 \mathrm{~h}$ at $4{ }^{\circ} \mathrm{C}$ with $160 \mu \mathrm{L}$ of AGE/ALEHSA at the concentration of $0.125 \mathrm{mg} / \mathrm{mL}$ in $20 \mathrm{mM}$ HEPES, $\mathrm{pH} 7.1,100 \mathrm{mM} \mathrm{NaCl}$ or with different concentrations in the same volume. After removal of the unbound fraction and three washes with $500 \mu \mathrm{L}$ of the same buffer, the elution was performed by boiling the beads in a 2x SDS sample buffer [0.125 M Tris-HCl, pH 6.8, 4\% w/v SDS, 200 mM dithiothreitol, 20\% (w/v) glycerol and $0.02 \%$ bromophenol Blue]. Samples were analyzed by SDS-PAGE and proteins were stained with Biosafe Coomassie (Bio-Rad). In the analysis, the densitometric data (A.u., Arbitrary unit) obtained from the bound material detected at high molecular weight ( $\geq 250 \mathrm{kDa}$ ) were summed with the A.u. obtained in the area around $70 \mathrm{kDa}$. Also the bands of VC1, p34 or p36 were analyzed by densitometry and used to normalize the data. The images were acquired by a Bio-Rad GS800 calibrated densitometer and the software Quantity One version 4.6.6 (Bio-Rad) was used for densitometry.

\section{Results}

\subsection{N-glycosylation of VC1 glycoforms secreted by P. pastoris}

As shown in Fig. 1A, each module of VC1 is a $\beta$-sandwich whose two facing $\beta$-sheets are held together by an intra-domain disulfide bridge buried in the protein core. The two domains are connected through hydrophobic interactions and hydrogen bonds [18, 25]. Both $N$-glycosylation sites are located in the $\mathrm{V}$ portion, being Asn25 in the $\beta$-strand $\mathrm{A}$ and Asn81 is in the loop connecting $\beta$-strand D with $\beta$-strand E (Fig. 1A) [18]. VC1 secreted by P. pastoris carries two Cterminal tags designed to facilitate purification and immobilize the hybrid protein on Streptavidin magnetic beads (Fig. 1A). The tagged VC1 is secreted in the medium as a doublet of VC1 variants named after their molecular mass p36 and p34 [19]. A previous peptide mapping analysis revealed that p36 derived from the full glycosylation of Asn25 and Asn81 whereas p34 resulted from the occupancy of just Asn25 [19]. In this work, the two variants were separated one from the other by a 
cation-exchange chromatography (Materials and methods) that improved the procedure previously described [19]. The fractions containing a single variant were pooled (Fig. S1) and then checked for purity by SDS-PAGE as shown in Fig. 1B. The p36 and p34 forms were used for the subsequent $N$ glycan analysis.

Isolated p36 and p34 were denatured and treated with peptide- $N$-glycosidase F (PNGase F) that releases the $N$-linked chains and converts asparagine into aspartic acid. The released $N$-glycans were labeled with procainamide according to a recently described highly sensitive derivatization method for glycan labeling and analyzed as described in Materials and methods [24]. In Fig. 2, the chromatograms of the oligosaccharides obtained from p36 and p34 are shown. The major detected species corresponded to $\mathrm{GlcNAc}_{2}-\mathrm{Hex}_{9-14}$. The chromatograms of p36 and of p34 were very similar but the most abundant species from p36 was GlcNAc $-\mathrm{Hex}_{10}$ whereas for p34 the most represented species was GlcNAc $-\mathrm{Hex}_{11}$. Table 1 shows that $\mathrm{GlcNAc}_{2}-\mathrm{Hex}_{10}$ represented $\sim 45 \%$ of the total 7 glycovariants from p36 whereas the GlcNAc $2-H_{11}$ was $~ 37 \%$ of the p34 glycovariants. Moreover, the size of these chains indicates that the $N$-glycans in VC1 are of the "core" type and are in good agreement with previously reported analyses of $P$. pastoris $N$-glycans [26, 27]. In addition, the mass of single peaks was compatible with the existence of different isomers. In general, the shape of the peaks, more symmetrical in p36 than in p34, indicates that the oligosaccharide chains from p36 were more homogeneous than the glycans obtained from p34. The experiment was repeated and the results were highly reproducible (data not shown).

In order to assess the composition of the $\mathrm{N}$-glycans, the oligosaccharides were subjected to exoglycosidase analysis. By use of the Jack Bean Mannosidase (JBM), a broad specificity mannosidase that removes $\alpha-1,2, \alpha-1,3$ and $\alpha-1,6$ linked mannose residues from the terminal ends, we determined that all the chains contained mannose except for one isomer present in traces and resistant to JBM that was detected both in p36 and p34 and could contain a terminal glucose or galactose or $\beta-1,2$ mannose (Fig. 3). While galactose is present in Schizosaccharomyces pombe $N$-glycans [28], to our knowledge it has never been reported in P. pastoris chains. Therefore, the JBM-resistant isomer may result from (i) a glucosidase II missing cleavage of the $\alpha-1,3$ linkage between the innermost glucose and the mannose at the terminal position of branch A of the $N$-linked oligosaccharide or (ii) from the presence of a $\beta$-1,2-linked mannose since $\beta$-1,2-Man has been reported in P. pastoris $N$ glycans and a family of $\beta$-mannosyltransferases genes was also identified in this yeast [29, 30]. In conclusion, the $N$-glycans of VC1 are of “core" type and "high mannose content". Microheterogeneity of oligosaccharides is higher in p34 than in p36. 


\subsection{Effect of the removal of N-glycans on the conformational stability of VC1}

To investigate the effects of the $N$-glycosylation on the secondary structure and on the overall conformational stability of VC1, CD studies were carried out on the glycosylated and the Endo Htreated protein. Deglycosylation in native conditions by PNGase F proved to be difficult. The enzyme completely removed the $N$-linked chains bound to Asn25 but only partially digested the chain linked to Asn81, suggesting that protein conformation around this site hampers the access of the enzyme to the polypeptide chain (data not shown). A complete removal was obtained by digesting the native protein with Endo $H$ that cleaves between the GlcNAc residues and leaves one GlcNAc bound to asparagine. The digestion of VC1 converted both forms into a single polypeptide of about $32 \mathrm{kDa}$ (p32) (Fig. 4A).

Far-UV CD spectra of the glycosylated and Endo H-treated proteins were almost superimposable (data not shown), suggesting that bound glycans do not affect the secondary structure of the VC1 domain. Overall conformational stability was assessed in temperature ramp experiments where unfolding was measured as change in the ellipticity at $205 \mathrm{~nm}$, as previously reported for $\beta 2$ microglobulin that also contains Ig-like domains [31]. Glycosylated and Endo $H$ treated proteins show very close unfolding curves from which the melting temperatures $(T \mathrm{~m})$ were determined. The $T_{\mathrm{m}}$ of the native VC1 was $54.5^{\circ} \mathrm{C}$ whereas $T_{\mathrm{m}}$ of the Endo H-treated VC1 was $53.3^{\circ} \mathrm{C}$ (Fig. 4B). Moreover, for both forms, denaturation was apparently monophasic with only one flex point in the curve that describes the transition from the native to the denatured state. This latter observation underlines the cooperative mutual interactions existing between the two domains, where the slightly stabilizing effects exerted by glycosylation are not restricted to the $\mathrm{V}$ domain, but are shared with the C1 domain. The recovery of the folded state after thermal denaturation, by reversing the temperature ramp, did not occur either for the glycosylated or the Endo H-treated VC1, suggesting that unfolding is an irreversible process and is not affected by the $N$-linked glycans (data not shown).

\subsection{Effects of VC1 glycosylation on AGEs and ALEs binding}

The impact $N$-glycans have on binding of VC1 to AGEs/ALEs was examined by testing VC1 binding capability after enzymatic removal of $N$-linked glycans in native conditions. Untreated or Endo H-treated VC1 were immobilized to streptavidin-coated beads through the Strep-tag and pull down assays were performed using AGE-HSA or ALE-HSA obtained by incubation of recombinant HSA ( 66 kDa) with ribose or MDA respectively, and previously characterized by MS analysis 
[17]. To exclude any effect of the 16 h-incubation at $37^{\circ} \mathrm{C}$ required for the enzymatic treatment of the protein, VC1 not incubated at $37^{\circ} \mathrm{C}$ was also immobilized in parallel. In addition, streptavidincoated beads represented a negative control.

Both glycosylated and Endo H-treated VC1 preferentially retained HMW-cross-linked species of AGE-HSA or ALE-HSA, as well as VC1 not incubated at $37{ }^{\circ} \mathrm{C}$ (Fig. 5A and B). These results suggest that glycans do not contribute to the binding of VC1 to AGEs/ALEs and that prolonged temperature incubation at $37^{\circ} \mathrm{C}$ does not affect the binding capacity of VC1. The control-beads did not retain any material indicating the absence of non-specific binding to the beads, in agreement with previous results [17].

Finally, to evaluate a possible mild effect of $N$-glycans on binding, we performed pull down assays in the presence of a fixed amount of VC1 immobilized to the beads and increasing concentrations of AGE-HSA the highest of which was under the saturating concentration of VC1. A representative gel and the corresponding graph show that the material bound to VC1 increases with the increase of the amount of AGE-HSA applied to the beads and the absence or presence of $N$-glycans do not influence VC1 binding property (Fig. 5C and D). The pull down assays were performed with the isolated p34 and p36 variants of VC1 and AGE-HSA or ALE-HSA. As shown in Fig. S2, no relevant differences were detected in the binding of the two VC1 variants to these ligands. Overall, these results suggest that the binding of AGEs and ALEs to VC1 does not depend on $N$-linked oligosaccharides.

\section{Discussion}

The interest toward RAGE and the role of AGEs and ALEs in human diseases is steadily increasing. In this work, we attempted to elucidate aspects of the ligand binding domain of RAGE, the VC1 domain, expressed as glycosylated protein in $P$. pastoris. We analyzed features that were not reported before for VC1 and, in particular, the characteristics of the glycan moiety, the effect of the carbohydrate moiety on protein stability and the influence of the presence or absence of $N$ glycans on binding of VC1 to AGEs and also to ALEs, being the latter a class of poorly-studied non enzymatic modifications of proteins that we recently reported to be in vitro RAGE ligands [17].

We determined that the $N$-linked chains from VC1 of $P$. pastoris are of the core-type and mannoserich and no hypermannosylation, that in Saccharomyces cerevisiae leads to outer chains of 100-150 mannose residues, was detected for VC1 [27]. While $N$-glycans from the two variants of VC1, p36 and p34, have the same size, their distribution varies, being the $\mathrm{Man}_{10} \mathrm{GlcNAc}_{2-}$ and 
$\mathrm{Man}_{11} \mathrm{GlcNAc}_{2}$ - the most abundant species in p36 and p34, respectively. This microheterogeneity of oligosaccharides is typical of $P$. pastoris [26, 27]. However, the $N$-linked chains appeared more homogeneous for p36 than for p34, indicating that $N$-linked chains at Asn25 in p34 are less mature than $N$-linked chains from Asn25 and Asn81 resulting from digestion of p36. The difference in abundance suggests that the occupancy status of Asn25 and Asn81 affects the processing of the $N$ linked chains that occurs in the Golgi.

The expression of mammalian glycoproteins or of single glycosylated domains in heterologous systems often poses problems of stability of the recombinant protein. Protein stability is affected by many factors and in secreted proteins major key players are the attainment of proper folding, the formation of the correct disulfide bonds in the ER (by oxidative folding), and the addition of $N$ oligosaccharide chains, a process that competes with and influences folding during the cotranslational translocation of the nascent polypeptide into the ER lumen [32, 33]. These processes are crucial during protein biosynthesis and $N$-glycosylation also promotes protein secretion. $N$ glycans may also affect the properties of the mature secreted product. To evaluate the contribution of the $N$-glycans on the conformational stability of the VC1 protein secreted by $P$. pastoris we used CD. The $N$-glycans did not affect VC1 protein conformation in unperturbed conditions and had a small stabilizing effect on the VC1 protein as evidenced by thermal denaturation experiments. This result suggests that once VC1 attains the proper structure, $N$-glycans are not essential for the conformational stability of the protein. However, since Endo H digestion leaves one GlcNac residue attached to the polypeptide chain we cannot exclude that a totally unglycosylated protein might have a lower stability. In addition, it is worth noticing that VC1 has four cysteine residues engaged in two disulfide bonds that may provide restraints to the dynamicity of the protein and contribute to protein stability.

Finally, in glycoproteins, the oligosaccharide chain extends into the solvent in a disorder conformation but the innermost sugar residues of a chain that occupies a site are more in contact with the local protein structure and may reduce solvent accessibility by steric hindrance. Therefore, we evaluated whether $N$-glycans could affect VC1 binding properties. Using the VC1 pull down as assay system and sugar-derived AGE-HSA or MDA-derived ALE-HSA as model ligands, no relevant differences in binding between the wild type VC1 and the Endo H-treated counterpart were detected. The inspection of the VC1 structure provides a possible explanation of this result since Asn25 and Asn81 are on the edges of the $\mathrm{V}$ domain and are located in opposite positions (Fig. 1A) Asn25 is in close proximity to the region of $\mathrm{V}$ that forms a highly positively charged convex surface 
to which AGE-serum albumins bind, whereas Asn81 is on the face where the hydrophobic region is present [18, 25, 34, 35].

It has been shown that G82S polymorphism of hRAGE leads to increased affinity for ligands, and increased signaling [22, 36-38]. These effects were proposed to be caused by complete glycosylation of Asn81 gained by the mutated receptor [22]. It is still controversial whether this polymorphism is associated with diseases since clinical studies revealed no association of G82S polymorphism with diabetic retinopathy in the Japanese Type 2 diabetic population [39] or with microangiopathy in the Chinese population [40] whereas a positive relation was detected with skin complications and nephropathy in Caucasian diabetic subjects [39]. At the protein structure level, the G82S polymorphism causes a local change of the V domain and a more global destabilization of the protein that likely makes N81 more accessible to the OST machinery responsible of the glycosylation in the ER [22, 34]. The increased affinity for AGE-BSA of the G82S V-domain produced in bacteria (unglycosylated), suggests that the change in protein conformation is the primary cause of the increased binding. Therefore, our results indicating that glycosylation of the wild type VC1 at Asn81 is not relevant for binding to AGE-HSA may be related to a more rigid conformation of the natural protein. The G82S replacement will be introduced in the VC1 from $P$. pastoris in order to deepen the study on the conformational stability of the mutated protein.

Profiling and defining the role of the $N$-glycans in binding can be beneficial in characterizing the properties of the affinity methods based on VC1 and their suitability for in vitro applications and in providing tools to rule out that VC1 may bind a potential protein partner through the glycan moiety. On the basis of the features described in this work, we propose VC1 from $P$. pastoris as a robust model protein for the set-up of enrichment strategies of RAGE ligands from different biological sources and therefore as a relevant reagent in the studies on this important human receptor.

The $N$-linked chains elaborated in the Golgi apparatus are different between lower eukaryotes and mammalian cells for composition and structure. sRAGE produced in CHO cells increased the in vivo bioactivity in a model system of arterial inflammation compared to the expression in insect cells [41]. Potential use of VC1 for medicine applications may require engineering of the glycan chains made in $P$. pastoris. This can be in principle obtained by engineering $N$-linked oligosaccharides through genetic intervention as reported in several studies aimed to humanize glycoproteins produced in P. pastoris although this is outside the purpose of our studies [42, 43].

\section{Acknowledgements}


This work was partially supported by University of Milan. G.D. is the recipient of a Postdoc fellowship from University of Milano. The authors wish to thank Euroclone S.p.A., Via Figino 20/22, Pero (Milano, Italy) that, as a partner of the CBM consortium (Connecting bio-research and industry), supported this work with the grant Art. 13 DM 593 08/08/2000 and in particular we are grateful to Dr. Fabio Bolchi for helpful discussions and continuous support. 


\section{Legends}

Fig 1. 3D-molecular model of VC1 and purification of p36 and p34. A. 3D-molecular model of VC1 domain of hRAGE was obtained using the software PyMOL and the PDB coordinates 3CJJ. The $\mathrm{V}$ domain is represented in blue (residues 23-119, numbering refers to the precursor of hRAGE), the C1 domain is in green (residues 120-233). Asn25 and Asn81 are in magenta. Disulfide bonds are in yellow (Cys38-Cys99 in the V domain and Cys144-Cys208 in the C1 domain). Tandem-tags separated by a spacer are present at the C-terminus of VC1 domain expressed in $P$. pastoris. B. Separation of the VC1 variants. VC1 was purified by Nickel-affinity chromatography from culture supernatant collected after $72 \mathrm{~h}$ of induction and subjected to a cationexchange chromatography in order to separate with a linear ionic-strength gradient the two glycoforms, p36 and p34 (Fig. S1). The figure shows the purified VC1 and the isolated p36 and p34 forms analyzed by SDS-PAGE and Coomassie blue staining.

Fig. 2. Chromatograms of procainamide-labeled glycans from p36 and p34. A. $N$-glycans released by Rapid PNGase F from p36 and purified by HILIC separation, were analyzed using a BEHXbridge column on a HPLC system coupled with a LTQ-Orbitrap Velos Mass spectrometer. B. Higher resolution of the species eluted and structure assignment in accordance with the exact masses detected in the MS channel. C. $N$-glycans released by Rapid PNGase F from p34 were analyzed as described for p36. D. Higher resolution of the species eluted and structure assignment in accordance with the exact masses detected in the MS channel.

Fig. 3. Structural analysis of $N$-glycans. Chromatograms showing p36 N-glycans (A, B) and p34 Nglycans (C, D) before and after treatment with JBM. After enzymatic digestion (B, D) all glycans were converted to small core mannose types, except for a low abundant species (RT 34.99 min and $34.98 \mathrm{~min})$ resistant to JBM. A possible structure consistent with the exact mass detected $(\mathrm{m} / \mathrm{z} \mathrm{H}+$ 1940.6) is shown. Hex indicates an hexose (glucose, galactose or $\beta-1,2$ mannose) that is not removed by JBM. The symbols are: - Mannose (Man); n N-Acetylglucosamine (GN); 0 Undetermined hexose (Hex).

Fig. 4. Thermal stability of glycosylated and Endo H-treated VC1. A. VC1 incubated for $16 \mathrm{~h}$ at 37 ${ }^{\circ} \mathrm{C}$ in the absence (-) or presence $(+)$ of Endo $\mathrm{H}$ in native conditions and VC1 not incubated at $37^{\circ} \mathrm{C}$ were analyzed by SDS-PAGE and Coomassie Blue staining. The enzymatic digestion of VC1 gives origin to a single $\sim 32 \mathrm{kDa}$-polypeptide (p32). B. Thermal stability of untreated (closed circle) or 
Endo H-treated (empty circle) VC1 at the concentration of $0.2 \mathrm{mg} / \mathrm{mL}$ in $50 \mathrm{mM}$ sodium phosphate buffer, pH 7.1. The fraction of folded protein was calculated from the changes in ellipticity at 205 nm.

Fig. 5. Effects of $N$-glycans on the binding of AGE-HSA or ALE-HSA to VC1. A. Pull down assays with AGE-HSA (20 $\mu \mathrm{g})$ and VC1 immobilized to Streptavidin coated magnetic beads. The input (IN, AGE-HSA) and the indicated fractions were analyzed by SDS-PAGE and Coomassie blue staining. Lanes refer to unbound or elution fractions from the following conjugated beads: 1 , VC1 not incubated at $37^{\circ} \mathrm{C}$; 2, VC1 incubated at $37^{\circ} \mathrm{C}$ without Endo $\mathrm{H}$; 3, VC1 incubated at 37 ${ }^{\circ} \mathrm{C}$ with Endo H ; 4: no VC1 (negative control). B, same as in A but the input (IN) is ALE-HSA. High MW species of AGE or ALE-HSA ( $\geq 250 \mathrm{kDa})$ are enriched by the VC1-pull down assays. No binding occurred with the control. In the image, VC1 and Endo H-treated VC1 polypeptides smear due to overload. The streptavidin band $(\sim 14 \mathrm{kDa})$ is also visible. C. SDS-PAGE and Coomassie blue staining of the elution fractions of pull down assays performed using beads with immobilized Endo H-treated (+) or untreated (-) VC1. As input, increasing amounts of AGE-HSA (2.5, 5, 7.5 and $10 \mu \mathrm{g}$ ) in a volume of $160 \mu \mathrm{l}$ were used. D. Relative quantification of the material bound in the pull down assay shown in panel $\mathrm{C}$. In the graph, the $\mathrm{Y}$-axis shows the ratio of the intensity of the AGE-HSA species and the intensity of the VC1 band present in the same bound material. The values of untreated or treated VC1 were corrected for the different staining with Coomassie Blue due to the presence or absence of the glycan chains. The corrected ratio values were plotted against the amount of AGE-HSA used as input.

\section{Supplementary material}

Fig. S1 Portion of an elution chromatogram of a cation exchange chromatography of purified VC1. The fractions 66-70 for p36 and 76-79 for p34 were pooled for further analyses.

Fig. S2 Binding of p34 and p36 to AGE-HSA and ALE-HSA. Pull down assays were performed with equal amount of p34 and p36 immobilized on beads and with the indicated concentrations of AGE-or ALE-HSA.

A. Bound material obtained with p34 or p36 from AGE-HSA (panel A) or ALE-HSA (panel C). The graphs in $B$ and $D$ show the ratio between the intensities of AGE-HSA or ALE-HSA species and the p34 or p36 bands present in the bound material of panels A and C, as a function of increasing amount of AGE- or ALEHSA present in the pull down. The values of p34 and p36 intensities were adjusted to correct for the different staining with Coomassie Blue due to the presence of 1 or 2 glycan chains. 
Table 1 . VC1 relative abundance of $N$-glycan peaks

\begin{tabular}{lccccccc}
\hline & \multicolumn{2}{c}{ VC1-p34 } & \multicolumn{2}{c}{ VC1-p36 } & \multicolumn{3}{c}{$\mathrm{m} / \mathrm{z}^{\mathrm{c}}$} \\
\cline { 2 - 7 } & RT $^{\mathrm{a}}$ & Area $^{\mathrm{b}}(\%)$ & RT $^{\mathrm{a}}$ & Area $^{\mathrm{b}}(\%)$ & $2 \mathrm{H}$ & $\mathrm{H}+\mathrm{Na}$ & $\mathrm{H}+\mathrm{K}$ \\
\hline Hex $_{9}$ GlcNAc $_{2}$ & 37.84 & 6.10 & 37.84 & 5.71 & 1052.0 & 1063.0 & 1070.6 \\
Hex $_{10}$ GlcNAc $_{2}$ & 39.49 & 24.86 & 39.49 & 44.74 & 1133.1 & 1144.0 & 1152.0 \\
Hex $_{11}$ GlcNAc $_{2}$ & 40.64 & 37.24 & 40.63 & 29.09 & 1214.1 & 1225.0 & 1233.1 \\
Hex $_{12}$ GlcNAc $_{2}$ & 41.51 & 19.17 & 41.49 & 13.10 & 1295.1 & 1303.5 & 1314.1 \\
Hex $_{13}$ GlcNAc $_{2}$ & 42.22 & 7.35 & 42.20 & 4.77 & 1376.1 & 1384.6 & 1395.4 \\
Hex $_{14}$ GlcNAc $_{2}$ & 42.83 & 3.74 & 42.82 & 1.82 & 1457.3 & 1464.6 & 1475.7 \\
Hex $_{15}$ GlcNAc $_{2}$ & 43.38 & 1.54 & 43.37 & 0.76 & 1538.7 & 1546.8 & N/D \\
\hline
\end{tabular}

Results are based on the areas under the peaks of the chromatograms in Fig. 2.

${ }^{\mathrm{a}}$ Retention time (min).

${ }^{b}$ The percentage indicates the proportion of the area of each peak over the total.

${ }^{\mathrm{c}}$ The last three columns show the distinctive signals observed for the indicated species. 


\section{References}

[1] G. Vistoli, D. De Maddis, A. Cipak, N. Zarkovic, M. Carini, G. Aldini, Advanced glycoxidation and lipoxidation end products (AGEs and ALEs): an overview of their mechanisms of formation, Free Radic Res, 47 Suppl 1 (2013) 3-27.

[2] C. Ott, K. Jacobs, E. Haucke, A. Navarrete Santos, T. Grune, A. Simm, Role of advanced glycation end products in cellular signaling, Redox Biol, 2 (2014) 411-429.

[3] Z.Q. Wang, L.L. Jing, J.C. Yan, Z. Sun, Z.Y. Bao, C. Shao, Q.W. Pang, Y. Geng, L.L. Zhang, L.H. Li, Role of AGEs in the progression and regression of atherosclerotic plaques, Glycoconjugate journal, (2018).

[4] A. Zhuang, J.M. Forbes, Diabetic kidney disease: a role for advanced glycation end-product receptor 1 (AGE-R1)?, Glycoconjugate journal, 33 (2016) 645-652.

[5] V.M. Monnier, N. Taniguchi, Advanced glycation in diabetes, aging and age-related diseases: conclusions, Glycoconjugate journal, 33 (2016) 691-692.

[6] R. Neviere, Y. Yu, L. Wang, F. Tessier, E. Boulanger, Implication of advanced glycation end products (Ages) and their receptor (Rage) on myocardial contractile and mitochondrial functions, Glycoconjugate journal, 33 (2016) 607-617.

[7] C.T. Raghavan, M. Smuda, A.J. Smith, S. Howell, D.G. Smith, A. Singh, P. Gupta, M.A. Glomb, I.M. Wormstone, R.H. Nagaraj, AGEs in human lens capsule promote the TGFbeta2-mediated EMT of lens epithelial cells: implications for age-associated fibrosis, Aging cell, 15 (2016) 465-476.

[8] N. Verzijl, J. DeGroot, Z.C. Ben, O. Brau-Benjamin, A. Maroudas, R.A. Bank, J. Mizrahi, C.G. Schalkwijk, S.R. Thorpe, J.W. Baynes, J.W. Bijlsma, F.P. Lafeber, J.M. TeKoppele, Crosslinking by advanced glycation end products increases the stiffness of the collagen network in human articular cartilage: a possible mechanism through which age is a risk factor for osteoarthritis, Arthritis Rheum, 46 (2002) 114-123.

[9] N. Verzijl, J. DeGroot, S.R. Thorpe, R.A. Bank, J.N. Shaw, T.J. Lyons, J.W. Bijlsma, F.P. Lafeber, J.W. Baynes, J.M. TeKoppele, Effect of collagen turnover on the accumulation of advanced glycation end products, J Biol Chem, 275 (2000) 39027-39031.

[10] S. Yamagishi, N. Nakamura, M. Suematsu, K. Kaseda, T. Matsui, Advanced Glycation End Products: A Molecular Target for Vascular Complications in Diabetes, Molecular medicine, 21 Suppl 1 (2015) S32-40.

[11] K. Fukami, S. Yamagishi, S. Okuda, Role of AGEs-RAGE system in cardiovascular disease, Curr Pharm Des, 20 (2014) 2395-2402.

[12] A. Bierhaus, P.M. Humpert, M. Morcos, T. Wendt, T. Chavakis, B. Arnold, D.M. Stern, P.P. Nawroth, Understanding RAGE, the receptor for advanced glycation end products, J Mol Med (Berl), 83 (2005) 876886.

[13] G. Aldini, G. Vistoli, M. Stefek, N. Chondrogianni, T. Grune, J. Sereikaite, I. Sadowska-Bartosz, G. Bartosz, Molecular strategies to prevent, inhibit, and degrade advanced glycoxidation and advanced lipoxidation end products, Free Radic Res, 47 Suppl 1 (2013) 93-137.

[14] S. Mizumoto, J. Takahashi, K. Sugahara, Receptor for advanced glycation end products (RAGE) functions as receptor for specific sulfated glycosaminoglycans, and anti-RAGE antibody or sulfated glycosaminoglycans delivered in vivo inhibit pulmonary metastasis of tumor cells, J Biol Chem, 287 (2012) 18985-18994.

[15] D. Xu, J. Young, D. Song, J.D. Esko, Heparan sulfate is essential for high mobility group protein 1 (HMGB1) signaling by the receptor for advanced glycation end products (RAGE), J Biol Chem, 286 (2011) 41736-41744.

[16] N. Rabbani, A. Ashour, P.J. Thornalley, Mass spectrometric determination of early and advanced glycation in biology, Glycoconjugate journal, 33 (2016) 553-568.

[17] G. Degani, A.A. Altomare, M. Colzani, C. Martino, A. Mazzolari, G. Fritz, G. Vistoli, L. Popolo, G. Aldini, A capture method based on the VC1 domain reveals new binding properties of the human receptor for advanced glycation end products (RAGE), Redox Biol, 11 (2017) 275-285.

[18] M. Koch, S. Chitayat, B.M. Dattilo, A. Schiefner, J. Diez, W.J. Chazin, G. Fritz, Structural Basis for Ligand Recognition and Activation of RAGE, Structure, 18 (2010) 1342-1352. 
[19] G. Degani, M. Colzani, A. Tettamanzi, L. Sorrentino, A. Aliverti, G. Fritz, G. Aldini, L. Popolo, An improved expression system for the VC1 ligand binding domain of the receptor for advanced glycation end products in Pichia pastoris, Protein Expr Purif, 114 (2015) 48-57.

[20] B.M. Dattilo, G. Fritz, E. Leclerc, C.W. Kooi, C.W. Heizmann, W.J. Chazin, The extracellular region of the receptor for advanced glycation end products is composed of two independent structural units, Biochemistry, 46 (2007) 6957-6970.

[21] T. Ostendorp, M. Weibel, E. Leclerc, P. Kleinert, P.M.H. Kroneck, C.W. Heizmann, G. Fritz, Expression and purification of the soluble isoform of human receptor for advanced glycation end products (sRAGE) from Pichia pastoris, Biochemical and Biophysical Research Communications, 347 (2006) 4-11.

[22] S.J. Park, T. Kleffmann, P.A. Hessian, The G82S polymorphism promotes glycosylation of the receptor for advanced glycation end products (RAGE) at asparagine 81: comparison of wild-type rage with the G82S polymorphic variant, J Biol Chem, 286 (2011) 21384-21392.

[23] H.T. Wright, Nonenzymatic deamidation of asparaginyl and glutaminyl residues in proteins, Critical reviews in biochemistry and molecular biology, 26 (1991) 1-52.

[24] A.J. Behrens, R.M. Duke, L.M. Petralia, D.J. Harvey, S. Lehoux, P.E. Magnelli, C.H. Taron, J.M. Foster, Glycosylation profiling of dog serum reveals differences compared to human serum, Glycobiology, 28 (2018) 825-831.

[25] H. Park, J.C. Boyington, The 1.5 A Crystal Structure of Human Receptor for Advanced Glycation Endproducts (RAGE) Ectodomains Reveals Unique Features Determining Ligand Binding, Journal of Biological Chemistry, 285 (2010) 40762-40770.

[26] R.B. Trimble, P.H. Atkinson, J.F. Tschopp, R.R. Townsend, F. Maley, Structure of oligosaccharides on Saccharomyces SUC2 invertase secreted by the methylotrophic yeast Pichia pastoris, J Biol Chem, 266 (1991) 22807-22817.

[27] T.R. Gemmill, R.B. Trimble, Overview of N- and O-linked oligosaccharide structures found in various yeast species, Biochim Biophys Acta, 1426 (1999) 227-237.

[28] F.D. Ziegler, T.R. Gemmill, R.B. Trimble, Glycoprotein synthesis in yeast. Early events in N-linked oligosaccharide processing in Schizosaccharomyces pombe, J Biol Chem, 269 (1994) 12527-12535.

[29] E. Vinogradov, B.O. Petersen, J.O. Duus, Isolation and characterization of non-labeled and 13C-labeled mannans from Pichia pastoris yeast, Carbohydrate research, 325 (2000) 216-221.

[30] C. Mille, P. Bobrowicz, P.A. Trinel, H. Li, E. Maes, Y. Guerardel, C. Fradin, M. Martinez-Esparza, R.C. Davidson, G. Janbon, D. Poulain, S. Wildt, Identification of a new family of genes involved in beta-1,2mannosylation of glycans in Pichia pastoris and Candida albicans, J Biol Chem, 283 (2008) 9724-9736.

[31] C. Santambrogio, S. Ricagno, M. Colombo, A. Barbiroli, F. Bonomi, V. Bellotti, M. Bolognesi, R. Grandori, DE-loop mutations affect beta2 microglobulin stability, oligomerization, and the low-pH unfolded form, Protein Sci, 19 (2010) 1386-1394.

[32] P.J. Robinson, M.A. Pringle, C.A. Woolhead, N.J. Bulleid, Folding of a single domain protein entering the endoplasmic reticulum precedes disulfide formation, J Biol Chem, 292 (2017) 6978-6986.

[33] J. Breitling, M. Aebi, N-linked protein glycosylation in the endoplasmic reticulum, Cold Spring Harbor perspectives in biology, 5 (2013) a013359.

[34] J. Xie, S. Reverdatto, A. Frolov, R. Hoffmann, D.S. Burz, A. Shekhtman, Structural basis for pattern recognition by the receptor for advanced glycation end products (RAGE), J Biol Chem, 283 (2008) 2725527269 .

[35] S. Matsumoto, T. Yoshida, H. Murata, S. Harada, N. Fujita, S. Nakamura, Y. Yamamoto, T. Watanabe, H. Yonekura, H. Yamamoto, T. Ohkubo, Y. Kobayashi, Solution structure of the variable-type domain of the receptor for advanced glycation end products: new insight into AGE-RAGE interaction, Biochemistry, 47 (2008) 12299-12311.

[36] S. Miller, A.P. Henry, E. Hodge, A.K. Kheirallah, C.K. Billington, T.L. Rimington, S.K. Bhaker, M. Obeidat, E. Melen, S.K. Merid, C. Swan, C. Gowland, C.P. Nelson, C.E. Stewart, C.E. Bolton, I. Kilty, A. Malarstig, S.G. Parker, M.F. Moffatt, A.J. Wardlaw, I.P. Hall, I. Sayers, The Ser82 RAGE Variant Affects Lung Function and Serum RAGE in Smokers and sRAGE Production In Vitro, PLoS One, 11 (2016) e0164041. 
[37] M.A. Hofmann, S. Drury, B.I. Hudson, M.R. Gleason, W. Qu, Y. Lu, E. Lalla, S. Chitnis, J. Monteiro, M.H. Stickland, L.G. Bucciarelli, B. Moser, G. Moxley, S. Itescu, P.J. Grant, P.K. Gregersen, D.M. Stern, A.M. Schmidt, RAGE and arthritis: the G82S polymorphism amplifies the inflammatory response, Genes and immunity, 3 (2002) 123-135.

[38] M. Osawa, Y. Yamamoto, S. Munesue, N. Murakami, S. Sakurai, T. Watanabe, H. Yonekura, Y. Uchigata, Y. Iwamoto, H. Yamamoto, De-N-glycosylation or G82S mutation of RAGE sensitizes its interaction with advanced glycation endproducts, Biochim Biophys Acta, 1770 (2007) 1468-1474.

[39] A. Kashiwagi, S. Araki, Relation between polymorphisms G1704T and G82S of RAGE gene and diabetic retinopathy in Japanese type 2 diabetic patients, Internal medicine, 44 (2005) 397-398.

[40] L. Liu, K. Xiang, RAGE Gly82Ser polymorphism in diabetic microangiopathy, Diabetes Care, 22 (1999) 646.

[41] H.J. Tae, J.M. Kim, S. Park, N. Tomiya, G. Li, W. Wei, N. Petrashevskaya, I. Ahmet, J. Pang, S. Cruschwitz, R.A. Riebe, Y. Zhang, C.H. Morrell, D. Browe, Y.C. Lee, R.P. Xiao, M.I. Talan, E.G. Lakatta, L. Lin, The Nglycoform of sRAGE is the key determinant for its therapeutic efficacy to attenuate injury-elicited arterial inflammation and neointimal growth, J Mol Med (Berl), 91 (2013) 1369-1381.

[42] S.R. Hamilton, R.C. Davidson, N. Sethuraman, J.H. Nett, Y. Jiang, S. Rios, P. Bobrowicz, T.A. Stadheim, H. Li, B.K. Choi, D. Hopkins, H. Wischnewski, J. Roser, T. Mitchell, R.R. Strawbridge, J. Hoopes, S. Wildt, T.U. Gerngross, Humanization of yeast to produce complex terminally sialylated glycoproteins, Science, 313 (2006) 1441-1443.

[43] B. Laukens, C. De Wachter, N. Callewaert, Engineering the Pichia pastoris N-Glycosylation Pathway Using the GlycoSwitch Technology, Methods Mol Biol, 1321 (2015) 103-122. 